\title{
Periodic solutions of second order difference systems with even potential
}

Huafeng Xiao*

\section{"Correspondence:}

huafeng@gzhu.edu.cn

School of Mathematics and

Information Science, Guangzhou

University, Guangzhou, China

Key Laboratory of Mathematics and

Interdisciplinary Science of

Guangdong, Guangzhou, China

\begin{abstract}
In this article, we study the multiplicity and minimality of periodic solutions to difference systems, which are globally superquadratic or subquadratic. A new technique is given to detect the minimal period of periodic solutions to autonomous systems. Some weaker conditions than a globally subquadratic condition are obtained to guarantee the existence of periodic solutions with prescribed minimal period to autonomous system.
\end{abstract}

Keywords: difference systems; periodic solution; minimal period; superquadratic; subquadratic

\section{Introduction}

On one hand, difference equations have been widely used to describe real-life situations in computer science, economics, neural network, ecology, cybernetics, etc. On the other hand, they are also natural consequences of the discretization of differential equations. So it is worthwhile to explore this topic. Many tools are used to study the existence of all kinds of solutions to discrete systems. A powerful tool is critical point theory, which firstly was introduced by Guo and Yu in 2003 to study the existence of periodic solutions to a difference system ( $c f$. [1-3]). Since then, the study of discrete dynamic systems has got considerable development. We refer to boundary value problems $(c f .[4,5])$, periodic solutions (cf. [6, 7]), homoclinic orbits $(c f .[8,9])$, and heteroclinic orbits $(c f .[10,11])$.

As is well known, the minimal periodic problem is an important but difficult problem. As far as the author knows, the study of solutions with a prescribed minimal period began in 2004. In that year, by estimating the energy of a variational functional, Yu et al. (cf. [12]) studied the existence of subharmonic solutions with a prescribed minimal period to a discrete forced pendulum equation. More recently, by making use of the Clark dual method, Bin (cf. [13]), Long (cf. [14]) and Long et al. (cf. [15]) studied the existence and multiplicity of periodic solutions with a prescribed minimal period to difference systems. Because of the lack of methods, results in this field are scarce.

It is well known that the Nehari manifold has been introduced by Nehari in 1960 ( $c f$. [16, 17]) and developed by Szulkin and Weth in 2010 (cf. [18]). It has been used widely to study the existence of ground state solutions to ordinary differential systems, partial differential systems, and difference systems ( $c f$. [19-21]). A ground state solution is a solution which possesses the minimal energy of all solutions. Since such a minimality can be used to prove

\section{黛 Springer}

○2014 Xiao; licensee Springer. This is an Open Access article distributed under the terms of the Creative Commons Attribution License (http://creativecommons.org/licenses/by/2.0), which permits unrestricted use, distribution, and reproduction in any medium, provided the original work is properly cited. 
the minimal periods of solutions, it has been used to study the existence of period solutions with prescribed minimal period to ordinary differential equations ( $c f$. [22-25]).

Motivated by the above references, in this paper, one attempts to make use of a Nehari manifold to study the multiplicity and minimality of periodic solutions to difference systems. When the systems are globally superquadratic or subquadratic, by restricting our discussion to the Nehari manifold, firstly, we study the existence of multiple periodic solutions to nonautonomous systems; secondly, we study the existence of periodic solutions with a prescribed minimal period to autonomous systems. Also, some subquadratic conditions, which are weaker than the globally subquadratic condition, are obtained to guarantee the existence of periodic solutions with prescribed minimal period to autonomous system.

For convenience, we denote by $\mathbb{N}, \mathbb{Z}, \mathbb{R}$ the sets of all natural numbers, integers, and real numbers, respectively. For $a, b \in \mathbb{Z}$ with $a \leq b$, define $Z[a, b]=\{a, a+1, \ldots, b\}$. For $m \in \mathbb{N}$, denote by $\mathbb{R}^{m}$ the Euclidean space with the usual inner product $(\cdot, \cdot)$ and norm $|\cdot|$.

Consider the nonautonomous difference system

$$
\triangle^{2} x_{n-1}+f\left(n, x_{n}\right)=0, \quad n \in \mathbb{Z},
$$

where $x_{n} \in \mathbb{R}^{m}, \triangle$ is a difference operator defined by $\Delta x_{n}=x_{n+1}-x_{n}$ and $\Delta^{2} x_{n}=\Delta\left(\Delta x_{n}\right)$.

Assume that:

(F1) there exists an even function $F \in C^{2}\left(\mathbb{R} \times \mathbb{R}^{m}, \mathbb{R}\right)$ such that $f(t, z)$ is the gradient of $F(t, z)$ with respect to $z$, i.e.,

$$
F(-t,-z)=F(t, z), \quad f(t, z)=\nabla_{z} F(t, z), \quad \forall(t, z) \in \mathbb{R} \times \mathbb{R}^{m} ;
$$

(F2) there exists a positive integer $T>0$ such that

$$
F(t+T, z)=F(t, z), \quad \forall(t, z) \in \mathbb{R} \times \mathbb{R}^{m}
$$

(F3) there exists a $\alpha>1$ such that

$$
0<\alpha(f(t, z), z) \leq\left(f^{\prime}(t, z) z, z\right), \quad \forall z \in \mathbb{R}^{m} \backslash\{0\}
$$

where $f^{\prime}(t, \cdot)$ denotes the Hermite matrix of $F(t, \cdot)$;

(F4) there exists a $\beta \in(0,1)$ such that

$$
0<\left(f^{\prime}(t, z) z, z\right) \leq \beta(f(t, z), z), \quad \forall z \in \mathbb{R}^{m} \backslash\{0\}
$$

Remark 1 If $F$ satisfies (F1), (F2), and (F3), without loss of generality, we can assume that $F(t, 0)=0$ for all $t \in \mathbb{R}$. Otherwise, there exists a twice continuously differentiable function $g(t)$ such that $F(t, 0)=g(t)$. Let $\widehat{F}(t, z)=F(t, z)-g(t)$. Then $\widehat{F}$ also satisfies (F1), (F2), and (F3) with $F$ replaced by $\widehat{F}$. Similarly, if $F$ satisfies (F1), (F2), and (F4), we can assume that $F(t, 0)=0$ for all $t \in \mathbb{R}$.

For a nonautonomous system, we have the following two results. 
Theorem 1 Suppose that $F$ satisfies (F1), (F2), and (F3). Then (1) has at least $m[(T-1) / 2]-$ 1 distinct pairs of different $T$-periodic solutions.

Theorem 2 Suppose that F satisfies (F1), (F2), and (F4). Then (1) has at least $m[(T-1) / 2]-$ 1 distinct pairs of different $T$-periodic solutions.

Consider the autonomous difference system

$$
\triangle^{2} x_{n-1}+f\left(x_{n}\right)=0, \quad n \in \mathbb{Z} .
$$

Assume that $F$ satisfies

(F5) there exists an even function $F \in C^{2}\left(\mathbb{R}^{m}, \mathbb{R}\right)$ such that $f(z)$ is the gradient of $F(z)$, i.e.,

$$
F(-z)=F(z), \quad f(z)=\nabla_{z} F(z), \quad \forall z \in \mathbb{R}^{m} ;
$$

(F6) there exists a $\alpha^{\prime}>1$ such that

$$
0<\alpha^{\prime}(f(z), z) \leq\left(f^{\prime}(z) z, z\right), \quad \forall z \in \mathbb{R}^{m} \backslash\{0\},
$$

where $f^{\prime}(\cdot)$ denotes the Hermite matrix of $F(\cdot)$;

(F7) there exists a $\beta^{\prime} \in(0,1)$ such that

$$
0<\left(f^{\prime}(z) z, z\right) \leq \beta^{\prime}(f(z), z), \quad \forall z \in \mathbb{R}^{m} \backslash\{0\} .
$$

Remark 2 If $F$ satisfies (F5) and (F6) (respectively, (F5) and (F7)), without loss of generality, we can assume that $F(0)=0$.

For an autonomous system, we have the following two results.

Theorem 3 Suppose that $F$ satisfies (F5) and (F6). Then, for any integer $P>1,(2)$ possesses at least a periodic solution with minimal period $P$.

Theorem 4 Suppose that $F$ satisfies (F5) and (F7). Then, for any integer $P>1,(2)$ possesses at least a periodic solution with minimal period $P$.

Now, one weakens the conditions (F5) and (F7). Assume that $F$ satisfies the following conditions:

(F8) there exists an even function $F \in C^{1}\left(\mathbb{R}^{m}, \mathbb{R}\right)$ such that $f(z)$ is the gradient of $F(z)$, i.e.,

$$
F(-z)=F(z), \quad f(z)=\nabla_{z} F(z), \quad \forall z \in \mathbb{R}^{m} ;
$$

(F9) there exist constants $G_{1}>0,0<\gamma<2$ such that for all $x \in \mathbb{R}^{m}$ and $|x| \geq G_{1}$,

$$
0<(f(x), x) \leq \gamma F(x)
$$


(F10) there exist constants $0<G_{2}<1, M_{1}>0$ and $0<\gamma^{\prime}<2$ such that for all $x \in \mathbb{R}^{m}$ and $|x|<G_{2}$,

$$
F(z) \geq M_{1}|z|^{\gamma^{\prime}}
$$

Remark 3 Assume that $F$ satisfies (F8) and (F9). Then there exist some positive constants $M_{2}$ and $M_{3}$ such that, for all $x \in \mathbb{R}^{m}$,

$$
F(x) \leq M_{2}|x|^{\gamma}+M_{3}
$$

Theorem 5 Suppose that $F$ satisfies (F8), (F9), and (F10). Then, for any integer $P>1$, (2) possesses at least a periodic solution with minimal period $P$.

The rest of this paper is divided into two parts. In Section 2, we study the multiplicity of periodic solutions to a nonautonomous system. In Section 3, firstly, we study the existence of periodic solutions with a prescribed minimal period to an autonomous system, which is globally superquadratic or subquadratic; secondly, some conditions, which are weaker than the globally subquadratic condition, are given to guarantee the existence of periodic solutions with prescribed minimal period to autonomous system.

\section{Nonautonomous difference system}

\subsection{Preparations}

By a similar argument to [2], we can build the space $E_{T}$

$$
E_{T}=\left\{x=\left(\ldots, x_{-n}, \ldots, x_{-1}, x_{0}, x_{1}, \ldots, x_{n}, \ldots\right) \mid x_{n+T}=x_{n}, x_{n} \in \mathbb{R}^{m}, \forall n \in \mathbb{Z}\right\} .
$$

$E_{T}$ is a Hilbert space equipping with the following norm and inner product

$$
\|x\|:=\left(\sum_{n=1}^{T}\left|x_{n}\right|^{2}\right)^{\frac{1}{2}}, \quad\langle x, y\rangle:=\sum_{n=1}^{T}\left(x_{n}, y_{n}\right), \quad \forall x, y \in E_{T} .
$$

It is easy to check that $E_{T}$ is linearly homeomorphic to $\mathbb{R}^{m T}$, which can also be identified with $\mathbb{R}^{m T}$.

For any $s>1$, we can also define $L^{s}$ norm on $E_{T}$ as follows:

$$
\|x\|_{s}:=\left(\sum_{n=1}^{T}\left|x_{n}\right|^{s}\right)^{\frac{1}{s}}
$$

Obviously, $\|\cdot\|=\|\cdot\|_{2}$. Since the space $E_{T}$ is a finite-dimensional space, all norms defined on it are equivalent. Hence, there exists a $C_{1, s}>1$ such that

$$
\frac{1}{C_{1, s}}\|x\|_{s} \leq\|x\| \leq C_{1, s}\|x\|_{s}, \quad \forall x \in E_{T} .
$$

The variational functional corresponding to (1) defined on $E_{T}$ is

$$
J(x)=\sum_{n=1}^{T}\left[\frac{1}{2}\left|\triangle x_{n}\right|^{2}-F\left(n, x_{n}\right)\right]=\frac{1}{2}\langle A x, x\rangle-\sum_{n=1}^{T} F\left(n, x_{n}\right),
$$


where $x=\left(x_{1}^{\tau}, x_{2}^{\tau}, \ldots, x_{T}^{\tau}\right)^{\tau}, A=P^{-1} C P, C=\operatorname{diag}(B, B, \ldots, B)_{m T \times m T}$. Here $\tau$ denotes the transposition of a vector and $B, P$ are matrices defined in [2].

Since $E_{T}$ is linearly homeomorphic to $\mathbb{R}^{m T}$, it follows from (F1) that $J$ can be viewed as a twice continuously differentiable functional defined on a finite-dimensional Hilbert space. Thus, for any $x, y, z \in E_{T}$, one has

$$
\begin{aligned}
& \left\langle J^{\prime}(x), y\right\rangle=\langle A x, y\rangle-\sum_{n=1}^{T}\left(f\left(n, x_{n}\right), y_{n}\right), \\
& \left\langle J^{\prime \prime}(x) z, y\right\rangle=\langle A z, y\rangle-\sum_{n=1}^{T}\left(f^{\prime}\left(n, x_{n}\right) z_{n}, y_{n}\right) .
\end{aligned}
$$

It is easy to check that the critical points of $J$ are $T$-periodic solutions of (1).

Similarly to reference [12], the eigenvalues of $B$ can be given by

$$
\lambda_{k}=4 \sin ^{2} \frac{k \pi}{T}, \quad k=0,1,2, \ldots, T-1 .
$$

By matrix theory, $A$ has $T$ eigenvalues $\lambda_{0}, \lambda_{1}, \ldots, \lambda_{T-1}$, each of multiplicity $m$. Clearly, 0 is an eigenvalue of $A$ and set $\eta=\left(\eta_{1}, \eta_{2}, \ldots, \eta_{m}\right)^{\tau}$ the eigenvector associated to 0 . If $T$ is even, $\lambda_{T / 2}=4$ is also an eigenvalue of $A$ and set $\eta^{\prime}=\left(\eta_{1}^{\prime}, \eta_{2}^{\prime}, \ldots, \eta_{m}^{\prime}\right)^{\tau}$, the eigenvector associated to $\lambda_{T / 2}$. For any $k \in Z[1,\lfloor(T-1) / 2\rfloor]$, where $\lfloor\cdot\rfloor$ denotes the upper integral function, set

$$
\eta_{n}^{k, 1}=a_{k} \cos \frac{2 k n \pi}{T}, \quad \eta_{n}^{k, 2}=b_{k} \sin \frac{2 k n \pi}{T}
$$

where $a_{k}, b_{k} \in \mathbb{R}^{m}$. Then $\eta_{n}^{k, 1}, \eta_{n}^{k, 2}$ are eigenvectors of $A$ corresponding to eigenvalue $\lambda_{k}$. Denote by $W_{i}(i=1,2,3,4)$ the spaces defined as follows:

$$
\begin{gathered}
W_{1}=\operatorname{span}\{\eta\}, \quad W_{2}=\operatorname{span}\left\{\eta_{n}^{k, 1}, k=1,2, \ldots,\left\lfloor\frac{T-1}{2}\right\rfloor\right\}, \\
W_{3}=\operatorname{span}\left\{\eta_{n}^{k, 2}, k=1,2, \ldots,\left\lfloor\frac{T-1}{2}\right\rfloor\right\}, \quad W_{4}=\operatorname{span}\left\{\eta^{\prime}\right\} .
\end{gathered}
$$

Then $\operatorname{dim}\left(W_{1}\right)=m, \operatorname{dim}\left(W_{2}\right)=\operatorname{dim}\left(W_{3}\right)=m\lfloor(T-1) / 2\rfloor$ and $\operatorname{dim}\left(W_{4}\right)=m$ if $T$ is even. Also, we have

$$
\begin{aligned}
& E_{T}=W_{1} \oplus W_{2} \oplus W_{3} \oplus W_{4}, \quad \text { when } T \text { is even; } \\
& E_{T}=W_{1} \oplus W_{2} \oplus W_{3}, \quad \text { when } T \text { is odd. }
\end{aligned}
$$

Thus, for any $x \in E_{T}, x_{n}$ has the form of

$$
\begin{aligned}
& x_{n}=a+\sum_{k=1}^{\frac{T}{2}-1}\left(a_{k} \cos \frac{2 k n \pi}{T}+b_{k} \sin \frac{2 k n \pi}{T}\right)+(-1)^{n} b, \quad \text { when } T \text { is even, } \\
& x_{n}=a+\sum_{k=1}^{\frac{T-1}{2}}\left(a_{k} \cos \frac{2 k n \pi}{T}+b_{k} \sin \frac{2 k n \pi}{T}\right), \quad \text { when } T \text { is odd, }
\end{aligned}
$$

where $a, b, a_{k}, b_{k}(k=1,2, \ldots,\lfloor(T-1) / 2\rfloor)$ are all constant vectors of $\mathbb{R}^{m}$. 
Now, we define a subspace of $E_{T}$ as follows:

$$
\widetilde{E}_{T}=\left\{x \in E_{T} \mid x_{n} \text { is odd in } n\right\} .
$$

Obviously, $\widetilde{E}_{T}=W_{3}$. Thus $\operatorname{dim}\left(\widetilde{E}_{T}\right)=m\lfloor(T-1) / 2\rfloor$. For any $x \in \widetilde{E}_{T}$, no matter $T$ is even or odd, $x_{n}$ has a Fourier expansion

$$
x_{n}=\sum_{k=1}^{\left\lfloor\frac{T-1}{2}\right\rfloor} a_{k} \sin \frac{2 k n \pi}{T} .
$$

Consider the following eigenvalue problem:

$$
A x=\lambda x, \quad x \in \widetilde{E}_{T}
$$

Problem (3) has $\lfloor(T-1) / 2\rfloor$ eigenvalues, each of them of multiplicity $m$. The eigenvalues of (3) $\operatorname{are} \lambda_{k}=4 \sin ^{2}(k \pi / T)$, where $k=1,2, \ldots,\lfloor(T-1) / 2\rfloor$. Obviously, the smallest eigenvalue is $\lambda_{1}$ and the largest eigenvalue is $\lambda_{\lfloor(T-1) / 2\rfloor}$, which is denoted by $\lambda_{\max }$.

Now we give a useful lemma. Since the proof is standard, we omit it.

Lemma 1 If $x$ is a critical point of $J$ restricted on $\widetilde{E}_{T}$, then $x$ is also a critical point of $J$ on $E_{T}$.

At the end of this subsection, two useful lemmas are given.

Suppose that $H$ be a real Banach space and $M$ is a closed symmetric $C^{1}$-submanifold of $H$ with $0 \notin M$. Suppose that $\phi \in C^{1}(M, \mathbb{R})$.

Lemma 2 [26] Suppose that $\phi$ is even and bounded below. Define

$$
c_{j}:=\inf _{A \in \Gamma_{j}} \sup _{x \in A} \phi(x), \quad j=1,2, \ldots
$$

where $\Gamma_{j}:=\{A \subset M: A=-A, A \subset H \backslash\{0\}, A$ is compact and $\gamma(A) \geq j\}$. If $\Gamma_{k} \neq \varnothing$ for some $k \geq 1$ and if $\phi$ satisfies the $(P S)_{c}$ for all $c=c_{j}, j=1,2, \ldots, k$, then $\phi$ has at least $k$ distinct pairs of critical points.

Lemma 3 [18] If $\phi$ is bounded below and satisfies the (PS) condition, then $c:=\inf _{M} \phi$ is attained and is a critical value of $\phi$.

\subsection{Superquadratic case}

In this subsection, we consider the existence of multiple periodic solutions of (1), where $F$ satisfies (F1), (F2), and (F3). Arguing similarly to [25], we can prove the following lemma.

Lemma 4 If $F$ satisfies (F1), (F2), and (F3), then $0<(1+\alpha) F(t, x) \leq(f(t, x), x)$ for all $x \in$ $\mathbb{R}^{m} \backslash\{0\}$. Also,

$$
F(t, x) \leq \bar{M}|x|^{\alpha+1}, \quad \text { when }|x| \leq 1, \quad F(t, x) \geq \underline{M}|x|^{\alpha+1} \quad \text { when }|x| \geq 1,
$$

where $\bar{M}=\max _{\{t \mid 0 \leq t \leq T\}} \max _{|x|=1} F(t, x)$ and $\underline{M}=\min _{\{t \mid 0 \leq t \leq T\}} \min _{|x|=1} F(t, x)$. 
Let $h(x)=\left\langle J^{\prime}(x), x\right\rangle$. Define a Nehari manifold $\mathcal{M}$ on $\widetilde{E}_{T}$ as follows:

$$
\mathcal{M}=\left\{x \in \widetilde{E}_{T} \backslash\{0\} \mid h(x)=0\right\}
$$

By a similar argument to reference [22], one can prove the following lemma.

Lemma $5 \mathcal{M}$ is $C^{1}$-manifold with dimension $m\lfloor(T-1) / 2\rfloor-1$. If $x_{0}$ is a critical point of $J$ restricted on $\mathcal{M}$, then $x_{0}$ is also a critical point of J restricted on $\widetilde{E}_{T}$.

By Lemmas 1 and 5, to search for periodic solutions of (1), we need find critical points of $J$ restricted on $\mathcal{M}$.

Lemma 6 Fixing $x \in \widetilde{E}_{T} \backslash\{0\}$, there exists a unique $t_{x}>0$ such that $t_{x} x \in \mathcal{M}$.

Proof Fixing $x \in \widetilde{E}_{T} \backslash\{0\}$, define $\varphi_{x}(t):=J(t x)$ for $t \in(0,+\infty)$. Obviously, $\varphi_{x} \in C^{2}$. It is easy to check that $\varphi_{x}^{\prime}(t)=0$ if and only if $t x \in \mathcal{M}$.

If $0<t<1 /\|x\|$ is small enough, then $\left|t x_{n}\right|<1$. It follows from Lemma 4 that $\left|F\left(n, t x_{n}\right)\right| \leq$ $\bar{M}\left|t x_{n}\right|^{\alpha+1}$. Thus

$$
\varphi_{x}(t)=\frac{1}{2}\langle t x, A(t x)\rangle-\sum_{n=1}^{T} F\left(n, t x_{n}\right) \geq \frac{t^{2}}{2} \lambda_{1}\|x\|^{2}-t^{1+\alpha} \bar{M} C_{1, \alpha+1}^{\alpha+1}\|x\|^{\alpha+1} .
$$

Since $\alpha>1$, there exists a $t_{1}>0$ depending only on $\|x\|$ such that

$$
\varphi_{x}(t)>\lambda_{1} t^{2}\|x\|^{2} / 4, \quad \forall t \in\left(0, t_{1}\right)
$$

Denote by $A_{0}(x)=\left\{n \in Z[1, T] \mid x_{n} \neq 0\right\}$. Obviously, $A_{0}(x) \neq \varnothing$. If $t>1 / \min \left\{\left|x_{n}\right| \mid n \in\right.$ $\left.A_{0}(x)\right\}$, then $t\left|x_{n}\right|>1$ for all $n \in A_{0}(x)$. It follows from Lemma 4 again that

$$
\begin{aligned}
\varphi_{x}(t) & =\frac{1}{2}\langle t x, A(t x)\rangle-\sum_{n=1}^{T} F\left(n, t x_{n}\right) \\
& \leq \frac{t^{2}}{2} \lambda_{\max }\|x\|^{2}-t^{1+\alpha} \underline{M} \sum_{n \in A_{0}(x)}\left|x_{n}\right|^{\alpha+1} \\
& \leq \frac{t^{2}}{2} \lambda_{\max }\|x\|^{2}-t^{1+\alpha} \underline{M} C_{1, \alpha+1}^{-(\alpha+1)}\|x\|^{\alpha+1} .
\end{aligned}
$$

Since $\alpha>1, \varphi_{x}(t)<0$ when $t>1$ is large enough. Thus the Rolle Mean Value Theorem implies that there exists a $t_{x}>0$ such that

$$
\varphi_{x}^{\prime}\left(t_{x}\right)=0
$$

Claim: There exists a unique $t_{x}>0$ satisfying (4).

Suppose, to the opposite, that there exist $0<t_{1}<t_{2}$ satisfying (4). By a direct computation, we have

$$
\varphi_{x}^{\prime}(t)=t\langle x, A x\rangle-\sum_{n=1}^{T}\left(f\left(n, t x_{n}\right), x_{n}\right), \quad \varphi_{x}^{\prime \prime}(t)=\langle x, A x\rangle-\sum_{n=1}^{T}\left(f^{\prime}\left(n, t x_{n}\right) x_{n}, x_{n}\right) .
$$


For $i=1,2$, since $\varphi_{x}^{\prime}\left(t_{i}\right)=0$, then $t_{i}\langle x, A x\rangle=\sum_{n=1}^{T}\left(f\left(n, t_{i} x_{n}\right), x_{n}\right)$. It follows from (F3) that

$$
\varphi_{x}^{\prime \prime}\left(t_{i}\right)=\frac{1}{t_{i}^{2}} \sum_{n=1}^{T}\left[\left(f\left(n, t_{i} x_{n}\right), t_{i} x_{n}\right)-\left(f^{\prime}\left(n, t_{i} x_{n}\right) t_{i} x_{n}, t_{i} x_{n}\right)\right]<0, \quad i=1,2
$$

Thus, there exists a $t_{3} \in\left(t_{1}, t_{2}\right)$ satisfying $\varphi_{x}\left(t_{3}\right)=\min _{t_{1} \leq t_{3} \leq t_{2}} \varphi_{x}(t)$. Consequently, $\varphi_{x}^{\prime}\left(t_{3}\right)=0$ and $\varphi_{x}^{\prime \prime}\left(t_{3}\right) \geq 0$. However, by a similar argument to $(5), \varphi_{x}^{\prime \prime}\left(t_{3}\right)<0$, which is a contradiction. Thus $t_{x}$ is unique.

Since $\varphi_{x}^{\prime}\left(t_{x}\right)=0$ and $\varphi_{x}^{\prime \prime}\left(t_{x}\right)<0$, then $\varphi_{x}\left(t_{x}\right)=\max _{t \in(0, \infty)} \varphi_{x}(t)$. Hence $J(t x)$ restricted on $(0, \infty)$ attains its maximum at $t_{x}$.

Lemma 7 satisfies the (PS) condition on $\mathcal{M}$.

Proof Assume that $\left\{x^{k}\right\} \subset \mathcal{M}$ is a (PS) sequence for $J$. Then there exists a $M_{4} \geq 0$ such that $\left|J\left(x^{k}\right)\right| \leq M_{4}$ for all $m \in \mathbb{N}$ and $J^{\prime}\left(x^{k}\right) \rightarrow 0$ as $k \rightarrow \infty$. Set

$$
A_{1}\left(x^{k}\right)=\left\{n \in Z[1, T]|| x_{n}^{k} \mid \leq 1\right\}, \quad A_{2}\left(x^{k}\right)=\left\{n \in Z[1, T]|| x_{n}^{k} \mid>1\right\} .
$$

Since $F$ is continuous, there exists a $M_{5}>0$ such that

$$
\left.|\underline{M}| x\right|^{1+\alpha}-F(t, x) \mid \leq M_{5}, \quad \forall t \in[0, T], x \in \mathbb{R}^{m} \text { with }|x| \leq 1 .
$$

Then

$$
\begin{aligned}
-M_{4} & \leq J\left(x^{k}\right)=\frac{1}{2}\left\langle x^{k}, A x^{k}\right\rangle-\sum_{n=1}^{T} F\left(n, x_{n}^{k}\right) \\
& \leq \frac{1}{2} \lambda_{\max }\left\|x^{k}\right\|^{2}-\sum_{n \in A_{1}\left(x^{k}\right)} F\left(n, x_{n}^{k}\right)-\underline{M} \sum_{n \in A_{2}\left(x^{k}\right)}\left|x_{n}^{k}\right|^{1+\alpha} \\
& \leq \frac{1}{2} \lambda_{\max }\left\|x^{k}\right\|^{2}+\sum_{n \in A_{1}\left(x^{k}\right)}\left[\underline{M}\left|x_{n}^{k}\right|^{1+\alpha}-F\left(n, x_{n}^{k}\right)\right]-\underline{M} C_{1,1+\alpha}^{-(1+\alpha)}\left\|x^{k}\right\|^{1+\alpha} \\
& \leq \frac{1}{2} \lambda_{\max }\left\|x^{k}\right\|^{2}+T M_{5}-\underline{M} C_{1,1+\alpha}^{-(1+\alpha)}\left\|x^{k}\right\|^{1+\alpha} .
\end{aligned}
$$

Thus $\underline{M} C_{1,1+\alpha}^{-(1+\alpha)}\left\|x^{k}\right\|^{1+\alpha}-1 / 2 \lambda_{\max }\left\|x^{k}\right\|^{2} \leq M_{4}+T M_{5}$. Since $\alpha>1,\left\{\left\|x^{k}\right\|\right\}$ is bounded. Since $\widetilde{E}_{T}$ is a finite-dimensional space, there exists a convergent subsequence of $\left\{x^{k}\right\}$.

Define a new map

$$
\begin{aligned}
& g: S^{1} \rightarrow \mathcal{M}, \\
& x \mapsto t_{x} x .
\end{aligned}
$$

It follows from Lemma 6 that $g$ is a bijection whose inverse $g^{-1}$ is given by $g^{-1}(x)=x /\|x\|$.

Lemma $8 C_{1}=\inf _{x \in \mathcal{M}} J(x)>0$. 
Proof For any $x \in \mathcal{M}$, since $J(x)=\sup _{t \in(0,+\infty)} J(t x)=\sup _{t \in(0,+\infty)} J(t x /\|x\|)$, it follows that

$$
\inf _{x \in \mathcal{M}} J(x)=\inf _{x \in \mathcal{M}} \sup _{t \in(0,+\infty)} J(t x)=\inf _{x \in S^{1}} \sup _{t \in(0,+\infty)} J(t x)
$$

To prove that $C_{1}>0$, one only need to show that $\inf _{x \in S^{1}} \sup _{t \in(0,+\infty)} J(t x)>0$.

Arguing similarly to Lemma 6 , there exists a $t_{4}>0$, which is independent of $x$, such that

$$
\varphi_{x}(t) \geq \lambda_{1} t^{2} / 4, \quad \forall 0<t<t_{4}, \forall x \in S^{1}
$$

Setting $t=t_{4} / 2$, one gets

$$
J\left(\frac{t_{4}}{2} x\right)=\varphi_{x}\left(\frac{t_{4}}{2}\right) \geq \frac{\lambda_{1} t_{4}^{2}}{16}>0, \quad \forall x \in S^{1} .
$$

Thus

$$
C_{1}=\inf _{x \in S^{1}} \sup _{t \in(0, \infty)} J(t x) \geq \frac{\lambda_{1} t_{4}^{2}}{16}>0 .
$$

Proof of Theorem 1 Because of (F2), $\mathcal{M}$ is a closed symmetric manifold and $0 \notin \mathcal{M}$. It follows from Lemma 5 that $\mathcal{M}$ is a $C^{1}$ manifold with dimension $m\lfloor(T-1) / 2\rfloor-1$. By Lemmas 7 and $8, J$ is bounded from below and satisfies the (PS) condition. It is easy to check that $J$ is even. Then Lemma 2 implies that $J$ has at least $m\lfloor(T-1) / 2\rfloor-1$ distinct pairs of critical points. Thus (1) possesses at least $m\lfloor(T-1) / 2\rfloor-1$ distinct pairs of periodic solutions.

\subsection{Subquadratic case}

In this subsection, we consider the multiplicity of periodic solutions of (1), where $F$ satisfies (F1), (F2), and (F4). In order to prove Theorem 2 , we consider the functional $J_{1}=-J$ and the Nehari manifold $\mathcal{M}_{1}$ is defined as $\mathcal{M}_{1}:=\left\{x \in \widetilde{E}_{T} \backslash\{0\} \mid\left\langle J_{1}^{\prime}(x), x\right\rangle=0\right\}$. Since the technique of the proof of Theorem 2 is just the same as that of Theorem 1, where $J$ and $\mathcal{M}$ are replaced by $J_{1}$ and $\mathcal{M}_{1}$, we omit it here.

\section{Autonomous difference equations}

\subsection{Variational framework}

In this section, we consider the existence of periodic solutions with any prescribed minimal period of (2), that is, for any given positive integer $P$, we search for periodic solutions of (2) with minimal period $P$.

The argument of this subsection is similar to that in Section 2.1. Let

$$
E_{P}=\left\{x=\left\{\ldots, x_{-n}, \ldots, x_{-1}, x_{0}, x_{1}, \ldots, x_{n}, \ldots\right\} \mid x_{n+P}=x_{n}, x_{n} \in \mathbb{R}^{m}, n \in \mathbb{Z}\right\},
$$

and equip $E_{P}$ with the inner product $\langle\cdot, \cdot\rangle_{P}$ as follows:

$$
\langle x, y\rangle_{P}=\sum_{n=1}^{P}\left(x_{n}, y_{n}\right), \quad \forall x, y \in E_{P} .
$$


Then $E_{P}$ is a Hilbert space, which is homeomorphic to $\mathbb{R}^{m P}$. Denote by $\|\cdot\|_{P}$ the norm introduced by $\langle\cdot, \cdot\rangle_{P}$.

Define the $L^{s}$ norm on $\widetilde{E}_{P}$ by

$$
\|x\|_{L^{s}}=\left(\sum_{n=1}^{P}|x|^{s}\right)^{\frac{1}{s}}
$$

Then $L^{s}$ is equivalent to $\|\cdot\|_{P}$ and there exists $C_{2, s}>0$ such that

$$
\frac{1}{C_{2, s}}\|x\|_{L^{s}} \leq\|x\|_{P} \leq C_{2, s}\|x\|_{L^{s}}, \quad \forall x \in \widetilde{E}_{P}
$$

The variational functional corresponding to (2) defined on $E_{P}$ is

$$
I(x)=\sum_{n=1}^{P}\left[\frac{1}{2}\left|\triangle x_{n}\right|^{2}-F\left(x_{n}\right)\right]=\frac{1}{2}\langle D x, x\rangle_{P}-\sum_{n=1}^{P} F\left(x_{n}\right),
$$

where \#D is a matrix of order $m p$. Then $I$ is twice continuously differentiable. The critical points of $I$ are $P$-periodic solutions of (2).

Define a subspace of $E_{P}$ as follows:

$$
\widetilde{E}_{P}=\left\{x_{n} \in E_{P} \mid x_{n} \text { is odd in } n\right\} .
$$

Then the eigenvalue problem

$$
D x=\lambda x, \quad x \in \widetilde{E}_{P}
$$

has $\lfloor(P-1) / 2\rfloor$ solutions, each of them of multiplicity $m$. The smallest eigenvalue is $\bar{\lambda}_{1}=$ $4 \sin ^{2}(\pi / P)$ and the largest eigenvalue is $\bar{\lambda}_{\lfloor(P-1) / 2\rfloor}$, which is denoted by $\bar{\lambda}_{\text {max }}$.

Lemma 9 If $x$ is a critical point of I restricted on $\widetilde{E}_{P}$, then $x$ is also a critical point of I on the whole space $E_{P}$.

At the end of this subsection, we define a Nehari manifold $\overline{\mathcal{M}}$ on $\widetilde{E}_{P}$ as follows:

$$
\overline{\mathcal{M}}=\left\{x \in \widetilde{E}_{P} \backslash\{0\} \mid\left\langle I^{\prime}(x), x\right\rangle_{P}=0\right\} .
$$

\subsection{Superquadratic case}

In this subsection, we assume that $F$ satisfies (F5) and (F6). The main target of this subsection is to prove Theorem 3. A similar argument to Section 2.2, one can check the following facts:

(i) $0<(1+\alpha) F(x) \leq(f(x), x)$ for all $x \in \mathbb{R}^{m} \backslash\{0\}$. Also,

$$
F(x) \leq \bar{M}^{\prime}|x|^{\alpha+1}, \quad \text { when }|x| \leq 1, \quad F(x) \geq \underline{M}^{\prime}|x|^{\alpha+1} \quad \text { when }|x| \geq 1,
$$

where $\bar{M}^{\prime}=\max _{|x|=1} F(x)$ and $\underline{M}^{\prime}=\min _{|x|=1} F(x)$;

(ii) $\overline{\mathcal{M}}$ is a $C^{1}$ manifold; 
(iii) critical points of $I$ restricted on $\overline{\mathcal{M}}$ are also critical points of $I$ restricted on $\widetilde{E}_{P}$;

(iv) for any $x \in \widetilde{E}_{P} \backslash\{0\}$, there exists a unique $t_{x}$ such that $t_{x} x \in \overline{\mathcal{M}}$ and

$$
I\left(t_{x} x\right)=\max _{t \in(0, \infty)} I(t x)
$$

(v) I restricted on $\overline{\mathcal{M}}$ satisfies the (PS) condition;

(vi) $I$ restricted on $\overline{\mathcal{M}}$ is bounded from below and $C_{2}=\inf _{x \in \overline{\mathcal{M}}} I(x)>0$.

Proof of Theorem 3 It follows from Lemma 3 that $C_{2}$ is a critical value. Denote by $\widetilde{x}$ the critical point of $I$ corresponding to $C_{2}$. Then $\widetilde{x}$ is a $P$-periodic solution of (2). It is easy to check that $\tilde{x}$ is a nonconstant periodic solution.

Claim: $\widetilde{x}$ has $P$ as its minimal period.

Suppose, to the opposite, that there exists a positive integer $k \geq 2$ such that $\tilde{x}$ has $P / k$ as its minimal period. Define $\widetilde{y}=\left\{\widetilde{y}_{l} \mid l \in Z[1, P]\right\}$ as follows:

$$
\tilde{y}_{l}=\widetilde{x}_{\left\lfloor\frac{l-1}{k}\right\rfloor+1} .
$$

Since $\tilde{x} \in \overline{\mathcal{M}}$, then $\tilde{y} \in \widetilde{E}_{P} \backslash\{0\}$. Thus there exists a $r_{\tilde{y}}>0$ such that $r_{\tilde{y}} \tilde{y} \in \overline{\mathcal{M}}$. Then

$$
\begin{aligned}
I\left(r_{\tilde{y}} \tilde{y}\right) & =\sum_{n=1}^{P}\left[\frac{1}{2}\left|\Delta r_{\hat{y}} \tilde{y}_{n}\right|^{2}-F\left(r_{\tilde{y}} \tilde{y}_{n}\right)\right] \\
& =\frac{1}{2} \sum_{n=1}^{P / k}\left|\Delta r_{\tilde{y}} \tilde{x}_{n}\right|^{2}-\sum_{n=1}^{P} F\left(r_{\tilde{y}} \tilde{x}_{n}\right) \\
& =\frac{1}{2 k} \sum_{n=1}^{P}\left|\Delta r_{\tilde{y}} \tilde{x}_{n}\right|^{2}-\sum_{n=1}^{P} F\left(r_{\tilde{y}} \tilde{x}_{n}\right) \\
& <\sum_{n=1}^{P}\left[\frac{1}{2}\left|\Delta r_{\tilde{y}} \tilde{x}_{n}\right|^{2}-F\left(r_{\tilde{y}} \tilde{x}_{n}\right)\right] \\
& =I\left(r_{\tilde{y}} \widetilde{x}\right) \leq I(\widetilde{x})=\inf _{x \in \overline{\mathcal{M}}} I(x) .
\end{aligned}
$$

This contradicts $r_{\tilde{y}} \tilde{y} \in \overline{\mathcal{M}}$. Hence $\tilde{x}$ has $P$ as its minimal period.

\subsection{Subquadratic case (I)}

In this subsection, we assume that $F$ satisfies (F5) and (F7). A similar argument to Section 2.2, we can prove the following facts:

(I) $0<(f(x), x) \leq\left(1+\beta^{\prime}\right) F(x)$ for all $x \in \mathbb{R}^{m} \backslash\{0\}$. Also,

$$
F(x) \geq \underline{M^{\prime}}|x|^{\beta^{\prime}+1}, \quad \text { when }|x| \leq 1, \quad F(x) \leq \bar{M}^{\prime}|x|^{\beta^{\prime}+1}, \quad \text { when }|x| \geq 1,
$$

where $\underline{M}^{\prime}, \bar{M}^{\prime}$ are defined in Section 3.2;

(II) $\overline{\mathcal{M}}$ is a $C^{1}$ manifold;

(III) critical points of $I$ restricted on $\overline{\mathcal{M}}$ are critical points of $I$ restricted on $\widetilde{E}_{P}$;

(IV) for any $x \in \widetilde{E}_{P} \backslash\{0\}$, there exists a unique $t_{x}$ such that $t_{x} x \in \overline{\mathcal{M}}$ and $I_{1}\left(t_{x} x\right)=\min _{t \in(0, \infty)} I_{1}(t x)$

(V) $I$ restricted on $\overline{\mathcal{M}}$ satisfies the (PS) condition. 
Lemma 10 Assume that $F$ satisfies (F5) and (F7). Then I restricted on $\widetilde{E}_{P}$ is bounded from below.

Proof It follows from Fact (I) that $F(x) \leq \bar{M}^{\prime}|x|^{\beta^{\prime}+1}$ when $|x| \geq 1$. Since $F$ is continuous, there exists a $M_{6}>0$ such that $F(x) \leq M_{6}$ when $|x| \leq 1$. Consequently, $F(x) \leq \bar{M}^{\prime}|x|^{\beta^{\prime}+1}+$ $M_{6}$ for all $x \in \mathbb{R}^{m}$. Thus

$$
\begin{aligned}
I(x) & =\frac{1}{2}\langle D x, x\rangle_{P}-\sum_{n=1}^{P} F\left(x_{n}\right) \geq \bar{\lambda}_{1}\|x\|_{P}^{2}-\sum_{n=1}^{P}\left[\bar{M}^{\prime}|x|^{\beta^{\prime}+1}+M_{6}\right] \\
& \geq \bar{\lambda}_{1}\|x\|_{P}^{2}-\bar{M}^{\prime} C_{2, \beta^{\prime}+1}^{\beta^{\prime}+1}\|x\|_{P}^{\beta^{\prime}+1}-P M_{6} .
\end{aligned}
$$

Since $\beta^{\prime} \in(0,1)$, there exists a constant $C_{3}$ such that $I_{1}(x) \geq C_{3}$ for all $x \in \widetilde{E}_{P}$.

Because of Lemma 10, $I$ restricted on $\overline{\mathcal{M}}$ is bounded from below. Denote by $C_{4}=$ $\inf _{x \in \overline{\mathcal{M}}} I(x)$. A similar argument to Lemma 8 , we can prove that $C_{4}<0$.

Proof of Theorem 4 It follows from Lemma 3 that $C_{4}$ is a critical value. Denote by $\widehat{x}$ the critical point of $I$ corresponding to $C_{4}$. Then $\widehat{x}$ is a nonconstant $P$-periodic solution of (2). By a similar discussion to the proof of Theorem 3 , we can prove that $\widehat{x}$ has $P$ as its minimal period.

\subsection{Subquadratic case (II)}

In this subsection, we assume that $F$ satisfies (F8), (F9), and (F10). By a similar argument to Lemma 10, we have the following lemma.

Lemma 11 Assume that $F$ satisfies (F8), (F9), and (F10). Then I restricted on $\widetilde{E}_{P}$ is bounded from below.

Lemma $12 C_{5}=\inf _{x \in \widetilde{E}_{P}} I(x)<0$.

Proof By (F10), $F(z) \geq M_{1}|z|^{\gamma^{\prime}}$ if $|x|<G_{2}$. Then, for any $x \in \widetilde{E}_{P}$ with $\|x\|_{P}<G_{2}$, we have

$$
\begin{aligned}
I(x) & =\frac{1}{2}\langle D x, x\rangle_{P}-\sum_{n=1}^{P} F\left(x_{n}\right) \leq \frac{1}{2} \bar{\lambda}_{\max }\|x\|_{P}^{2}-\sum_{n=1}^{P}\left[M_{1}\left|x_{n}\right|^{\gamma^{\prime}}\right] \\
& \leq\|x\|_{P}^{\gamma^{\prime}}\left[\frac{1}{2} \bar{\lambda}_{\max }\|x\|_{P}^{2-\gamma^{\prime}}-M_{1} C_{3, \gamma^{\prime}}^{-\gamma^{\prime}}\right] .
\end{aligned}
$$

Since $0<\gamma^{\prime}<2$, if $0<\|x\|_{P}<\min \left\{G_{2},\left[M_{1} C_{3, \gamma^{\prime}}^{-\gamma^{\prime}}\left[\bar{\lambda}_{\max }\right]^{1 /\left(2-\gamma^{\prime}\right)}\right\}\right.$, then

$$
I(x)<-\frac{\left(M_{1} C_{3, \gamma^{\prime}}^{-\gamma^{\prime}}\right)^{2 /\left(2-\gamma^{\prime}\right)}}{2\left(\bar{\lambda}_{\max }\right)^{1 /\left(2-\gamma^{\prime}\right)}} .
$$

Hence $C_{5}=\inf _{x \in \widetilde{E}_{P}} I(x)<0$. 
Lemma 13 I restricted on $\widetilde{E}_{P}$ satisfies the (PS) condition.

Proof Assume that $\left\{x^{k}\right\} \subset \widetilde{E}_{P}$ is a (PS) sequence for $I$. Then there exists a $M_{7} \geq 0$ such that $\left|I\left(x^{k}\right)\right| \leq M_{7}$ for all $k \in \mathbb{N}$ and $I^{\prime}\left(x^{k}\right) \rightarrow 0$ as $k \rightarrow \infty$. Then

$$
\begin{aligned}
M_{7} & \geq I\left(x^{k}\right)=\frac{1}{2}\left\langle x^{k}, D x^{k}\right\rangle-\sum_{n=1}^{P} F\left(x_{n}^{k}\right) \\
& \geq \frac{1}{2} \bar{\lambda}_{1}\left\|x^{k}\right\|^{2}-\sum_{n=1}^{P}\left(M_{2}\left|x^{k}\right|^{\gamma}+M_{3}\right) \\
& \geq \frac{1}{2} \bar{\lambda}_{1}\left\|x^{k}\right\|^{2}-M_{2} C_{2, \gamma}^{\gamma}\left\|x^{k}\right\|^{\gamma}-M_{3} P .
\end{aligned}
$$

Thus $1 / 2 \bar{\lambda}_{1}\left\|x^{k}\right\|^{2}-M_{2} C_{2, \gamma}^{\gamma}\left\|x^{k}\right\|^{\gamma} \leq M_{3} P+M_{7}$. Since $\gamma<2$, it follows that $\left\{x_{k}\right\}$ is bounded. Since $\widetilde{E}_{T}$ is a finite-dimensional space, there exists a convergent subsequence of $\left\{x^{k}\right\}$.

Proof of Theorem 5 Let $\left\{x^{k}\right\} \subset \widetilde{E}_{P}$ be a minimal sequence of $I$, that is, $I\left(x^{k}\right) \rightarrow C_{5}$ as $k \rightarrow \infty$. By the Ekeland variational principle, $I^{\prime}\left(x^{k}\right) \rightarrow 0$ as $k \rightarrow \infty$. Since $I$ satisfies the (PS) condition, $C_{5}$ is a critical point of $I$ restricted on $\widetilde{E}_{P}$. Denote by $\bar{x}$ the critical point of $I$ corresponding to critical value $C_{5}$. Then $\bar{x}$ is a periodic solution of (2). It is easy to check that $\bar{x}$ is a nonconstant $P$-periodic solution. By a similar discussion to the proof of Theorem 3 , we can prove that $\bar{x}$ has $P$ as its minimal period.

\section{Competing interests}

The author declares that he has no competing interests.

\section{Acknowledgements}

This project is supported by NSFC (No. 11126063).

Received: 24 March 2014 Accepted: 11 July 2014 Published: 04 Aug 2014

\section{References}

1. Guo, ZM, Yu, JS: Existence of periodic and subharmonic solutions for second-order superlinear difference equations. Sci. China Ser. A 46, 506-515 (2003)

2. Guo, ZM, Yu, JS: Existence of periodic and subharmonic solutions to subquadratic second-order equations. J. Lond. Math. Soc. 68, 419-430 (2003)

3. Guo, ZM, Yu, JS: Periodic and subharmonic solutions for superquadratic discrete Hamiltonian systems. Nonlinear Anal. 55, 969-983 (2003)

4. Tian, Y, Du, ZJ, Ge, WG: Existence results for discrete Sturm-Liouville problem via variational methods. J. Differ. Equ. Appl. 13, 467-478 (2007)

5. Yu, JS, Guo, ZM: Boundary value problems of discrete generalized Emden-Foeler equation. Sci. China Ser. A 49, 1303-1314 (2006)

6. Mawhin, J: Periodic solutions of second order nonlinear difference systems with $\phi$-Laplacian: a variational approach. Nonlinear Anal. 75, 4672-4687 (2012)

7. Zhang, $\mathrm{H}, \mathrm{Li}, \mathrm{ZX}$ : Periodic solutions for a class of nonlinear discrete Hamiltonian systems via critical point theory. J. Differ. Equ. Appl. 16, 1381-1391 (2010)

8. Lin, XY, Tang, XH: Existence of infinitely many homoclinic orbits in discrete Hamiltonian systems. J. Math. Anal. Appl. 373, 59-72 (2011)

9. Ma, MJ, Guo, ZM: Homoclinic orbits for second order self-adjoint difference equations. J. Math. Anal. Appl. 323, 513-521 (2006)

10. Xiao, HF, Yu, JS: Heteroclinic orbits for a discrete pendulum equation. J. Differ. Equ. Appl. 17, 1267-1280 (2011)

11. Zhang, H, Li, ZX: Heteroclinic orbits and heteroclinic chains for a discrete Hamiltonian system. Sci. China Ser. A 53, 1555-1564 (2010)

12. Yu, JS, Long, YH, Guo, ZM: Subharmonic solutions with prescribed minimal period of a discrete forced pendulum equation. J. Dyn. Differ. Equ. 16, 575-586 (2004)

13. Bin, HH: Subharmonics with minimal periods for convex discrete Hamiltonian systems. Abstr. Appl. Anal. 2013, 508247 (2013)

14. Long, YH: Applications of Clark duality to periodic solutions with minimal period for discrete Hamiltonian systems. J. Math. Anal. Appl. 342, 726-741 (2008) 
15. Long, YH, Guo, ZM, Shi, HP: Multiple periodic solutions with minimal period for second order discrete system. Acta Appl. Math. 110, 181-191 (2010)

16. Nehari, Z: On a class of nonlinear second-order differential equations. Trans. Am. Math. Soc. 95, 101-123 (1960)

17. Nehari, Z: Characteristic values associated with a class of nonlinear second order differential equations. Acta Math. $105,141-175(1961)$

18. Szulkin, A, Weth, T: The method of Nehari manifold. In: Handbook of Nonconvex Analysis and Applications. International Press, Somerville (2010)

19. Pankov, A: Periodic nonlinear Schrödinger equation with application to photonic crystals. Milan J. Math. 73, 259-287 (2005)

20. Pankov, A: On decay of solutions to nonlinear Schrödinger equations. Proc. Am. Math. Soc. 136, 2565-2570 (2008)

21. Szulkin, A, Weth, T: Ground state solutions for some indefinite variational problems. J. Funct. Anal. 257, $3802-3822$ (2009)

22. Ambrosetti, A, Mancini, G: Solutions of minimal period for a class of convex Hamiltonian systems. Math. Ann. 255 405-421 (1981)

23. Deng, ST: Minimal periodic solutions of a class of Hamiltonian systems. Acta Math. Sin. 27, 664-675 (1984) (in Chinese)

24. Magrone, P: Critical point methods for indefinite nonlinear Elliptic equations and Hamiltonian systems. PhD thesis, Universitá Degli Studi Di Roma 'Tor Vergata' (2001)

25. Xiao, YM: Periodic solutions with prescribed minimal period for the second order Hamiltonian systems with even potentials. Acta Math. Sin. Engl. Ser. 26, 825-830 (2010)

26. Szulkin, A: Ljusternik-Schnirelmann theory on $C^{1}$-manifold. Ann. Inst. Henri Poincaré, Anal. Non Linéaire 5, 119-139 (1988)

10.1186/1687-1847-2014-215

Cite this article as: Xiao: Periodic solutions of second order difference systems with even potential. Advances in Difference Equations 2014, 2014:215

\section{Submit your manuscript to a SpringerOpen ${ }^{\circ}$ journal and benefit from:}

- Convenient online submission

- Rigorous peer review

Immediate publication on acceptance

- Open access: articles freely available online

- High visibility within the field

- Retaining the copyright to your article 